\title{
EXAME PARASITOLÓGICO QUANTITATIVO DAS FEZES: ESTUDO COMPARATIVO ENTRE OS MÉTODOS DE McMASTER, STOLL-HAUSHEER E KATO-KATZ*
}

\author{
Vera Lúcia Pagliusi Castilho, Eliana Guizelini, Eliana Salgado Turrl, \\ Rubens Campos, Vicente Amato Neto, Antônio Augusto Baillot \\ Moreira e Pedro Luiz Silva Pinto
}

Foram comparados 300 exames parasitológicos quantitativos das fezes, pelos métodos de Kato-Katz, Stoll-Hausheer e McMaster, após análise prévia e rotineira,

Os resultados evidenciaram as porcentagens de positividades a seguir especificadas, levando em conta, por ordem, espécie de helminto, número de situafões nas quais os ovos respectivos apareceram e as participacões dos processos de Kato-Katz, StollHausheer e McMaster: ancilostomideos - 117 (89,7\%, 76,0\% e 80,3\%); Trichocephalus trichiurus-124(91,1\%, 70,9\% e 70,9\%); Ascaris lumbricoides-59(94,9\%, 84,7\% e 49,1\%); Schistosoma mansoni - 58 (96,5\%, 48,2\% e 0,0\%); Hymenolepis nana- 15 $(46,6 \%, 73,3 \%$ e $86,6 \%)$.

Quanto à sensibilidade dos métodos, a avaliação estatística demonstrou, na maioria das situações, vantagem do de Kato-Katz sobre os demais.

O estudo efetuado comprovou superioridade da técnica de Kato-Katz, em virtude de verificaçóes relacionadas com especificidade, sensibilidade e facilidade para execucão. O procedimento de McMaster não merece recomendação para uso habitual, uma vez que não é eficiente no que concerne à demonstração de ovos pesados e propicia preparações dificilmente examináveis.

Palavras-chaves: Exame parasitológico quantitativo das fezes. Estudo comparativo. Métodos de Kato-Katz, Stoll-Hausheer e McMaster.

A contagem, em matéria fecal, dos ovos de diversos helmintos intestinais é parâmetro de valor incontestável, como indice da intensidade de parasitoses e recurso laboratorial de avaliação da eficácia de procedimentos terapêuticos.

Os métodos de Stoll-Hausheer, Kato-Katz e McMaster são empregados com tais objetivos, mas cada um deles envolve indicações preferenciais diante das diversas espécies de vermes. A técnica de KatoKatz é de eleição no que concerne aos ovos de Schistosoma mansoni, a de Stoll-Hausheer tradicionalmente merece preferência para quantificar in-

\footnotetext{
* Trabalho do Laboratório de Investigação Médica - Parasitologia do Hospital das Clínicas da Faculdade de Medicina da Universidade de São Paulo.

Endereço para correspondência: Dr. Vicente Amato Neto, Laboratório de Investigação Médica - Parasitologia, Avenida Doutor Arnaldo 455, CEP 01246, São Paulo, Brasil. Recebido para publicação em 5/10/83.
}

fecção ancilostomótica e a de McMaster apenas tem tido aplicação, até agora, em Medicina Veterinária.

Estudos comparativos entre diversas técnicas coprológicas, aptas a determinaçōes quantitativas, têm contado com a atuação de vários pesquisadores $^{2} 34568910$ e esses trabalhos procuram ressaltar méritos referentes à especificidade quanto aos diversos parasitos e à sensibilidade em revelar maior número de ovos, como também preocupam-se com a influência de outros fatores, representados sobretudo por facilidade de execução, baixo custo e capacidade de refletir, da maneira mais expressiva possivel, intensidade de um processo, em termos individuais e populacionais.

No presente trabalho são comparadas três técnicas, conhecidas como de Kato-Katz, Stoll-Hausheer e McMaster, frente a enteroparasitos bastante prevalentes no ambiente onde exercemos atividades profissionais. Representaram objetivos básicos da investigação determinar a especificidade de cada um dos meios citados e a capacidade deles no sentido de confirmar maiores taxas numéricas nas contagens. 
Castilho VLP, Guizelini E, Turri ES, Campos R, Amato Neto V, Moreira AAB, Pinto PLS. Exame parasitológico quantitativo das fezes: estudo comparativo entre os métodos de McMaster, Stoll-Hausheer e Kato-Katz. Revista da Sociedade Brasileira de Medicina Tropical 17:209-212, Out-Dez, 1984

\section{MATERIAL E MÉTODOS}

Foram examinadas, para análise comparativa, 300 amostras de fezes encaminhadas rotineiramente à Seção de Parasitologia do Laboratório Central do Hospital das Clínicas da Faculdade de Medicina da Universidade de São Paulo, sendo 150 escolhidas ao acaso e outras 150 contendo seguramente ovos de helmintos, conforme avaliação prévia por centrífugoflutuação em sulfato de zinco e sedimentação espontânea em água. As diretrizes respeitadas para execução do processo de Stoll-Hausheer estão consignadas em compêndio especializado ${ }^{1}$; as atinentes ao de Kato-Katz encontram-se em publicação do Instituto de Pesquisas Johnson \& Johnson de Doenças Endêmicas, convindo frisar que as procuras de ovos de ancilostomideos e de Hymenolepis nana sucederam 30 minutos após o preparo da lâmina; quanto ao de McMaster, foi seguida metodologia descrita por Gordon e Whitlock ${ }^{6}$ e detalhada nesta oportunidade porque, parece-nos, não foi alvo ainda de apropriada divulgaçăo na área médica como ocorre na veterinária; a) homogeneizar as fezes e usar $2 \mathrm{ml}$, estipulados em seringa para injeção (recorrer a volume constitui modificação da conduta original, que se baseia em pesagem; a mudança, porém, é tradicionalmente aceita, uma vez que facilita o trabalho rotineiro); b) diluir em $28 \mathrm{ml}$ de solução saturada de cloreto de sódio, utilizando homogeneizador de acrilico; c) filtrar em coador e gaze dobrada quatro vezes, para melhor reter resíduos; d) por intermédio de contagotas, retirar parte do material filtrado, após homo- geneização, a fim de preencher a câmara de McMaster, que é composta por duas lâminas separadas por espaço de $1,5 \mathrm{~mm}$, entre os quais localiza-se um par de áreas com $1 \mathrm{~cm}^{2}$, sendo de $0,15 \mathrm{ml}$ o volume liquido de cada uma; e) aguardar dois minutos depois do preenchimento, de modo a esperar que os ovos flutuem, permanecendo em mesmo plano; f) efetuar exame microscópico, com aumento de $100 \mathrm{X}$; g) praticar duas contagens, calcular a média aritmética e multiplicar por 100 para estabelecer o número de ovos por $1 \mathrm{~g} \mathrm{de}$ matéria fecal*.

Os números registrados nessas avaliações quantitativas e pertinentes a cada um dos métodos sofreram análise estatística, mediante a aplicação do teste de Friedman, para verificar diferenças significantes entre os recursos laboratoriais focalizados, havendo a seguir emprego da prova múltipla, com a finalidade de ordenar as mesmas 5 .

\section{RESULTADOS}

Das 300 amostras examinadas, 240 possibilitaram comparações, por revelarem-se positivas em pelo menos uma das três técnicas de contagem.

Os resultados acerca da especificidade figuram na Tabela 1 , da qual consta $o$ número total de exames positivos para cada modalidade de helminto, derivado, no mínimo, de um dos procedimentos, ao lado das constatações e porcentagens tocantes aos três métodos cotejados ou adotados rotineiramente.

Tabela 1-Estudo comparativo entre métodos para exame parasitológico quantitativo das fezes: positividades conforme as técnicas utilizadas e os espécies de helmintos.

\section{Métodos}

\begin{tabular}{lrrrrr} 
Espécies de helmintos & Totais & Kato-Katz & $\begin{array}{c}\text { Stool- } \\
\text { Hausheer }\end{array}$ & McMaster & $\begin{array}{c}\text { Utilizados } \\
\text { prévia e } \\
\text { rotineira- } \\
\text { mente* }\end{array}$ \\
\hline Ancilostomídeos & 117 & $105(89,7 \%)$ & $89(76,0 \%)$ & $94(80,3 \%)$ & $102(87,1 \%)$ \\
Trichocephalus trichiurus & 124 & $113(91,1 \%)$ & $88(70,9 \%)$ & $88(70,9 \%)$ & $107(86,2 \%)$ \\
Ascaris lumbricoides & 59 & $56(94,9 \%)$ & $50(84,7 \%)$ & $29(49,1 \%)$ & $51(86,4 \%)$ \\
Schistosoma mansoni & 58 & $56(96,5 \%)$ & $28(48,2 \%)$ & $0(0,0 \%)$ & $32(53,4 \%)$ \\
Hymenolepis nana & 15 & $7(46,6 \%)$ & $11(73,3 \%)$ & $13(86,6 \%)$ & $13(86,6 \%)$ \\
\hline
\end{tabular}

* Centrífugo-flutuação no sulfato de zinco e sedimentação espontânea em água.

* O homogeneizador de acrilico e a câmara de McMaster são comercializados pela firma "Unilab" (Avenida Doutor Gentil de Moura, 277 - São Paulo, Estado de São Paulo).
A propósito da sensibilidade, as comprovações, nas contagens em cada material, evidenciaram após processamento estatístico: 
Castilho VLP, Guizelini E, Turri ES, Campos $R$, Amato Neto V, Moreira AAB, Pinto PLS. Exame parasitologico quantitativo das fezes: estudo comparativo entre os métodos de McMaster, Stoll-Hausheer e Kato-Katz. Revista da Sociedade Brasileira de Medicina Tropical 17:209-212, Out-Dez, 1984

- Para ovos de $H$. nana, o teste de Friedman $\left(x_{r}^{2}=3,1\right)$ não demonstrou diferenças significativas a nivel de 5,0\%;

Para ovos de Ascaris lumbricoides e $S$. mansoni, houve rejeição da hipótese de equivalência dos processos a nivel de significância menor que $0,1 \%$, com o valores de $\mathrm{x}_{\mathrm{r}}^{2}$ respectivamente de 59,6 e 59,7 ; a prova de comparação múltipla situou as técnica adotadas de acordo com os seguintes "postos": A. lumbricoides - Kato-Katz $=152,5$; Stoll-Hausheer $=$ 119 e McMaster $=70,5$, sendo a menor diferença para que elas possam ser escalonadas $(\mathrm{c}=25,5) ; S$. mansoni - Kato-Katz $=160$, Stoll-Hausheer $=118$; McMaster $=76(\mathrm{c}=26,0)$.

Para Trichocephalus trichiurus, o teste de Friedman detectou diferença significativa a nivel de $0,1 \%\left(x_{r}^{2}=62,1\right) ;$ do de comparação múltipla decorreu o seguinte: Kato-Katz $=317,5$, Mc Master $=208$, Stoll-Hausheer $=207(c=37,4)$.

Para ovos de ancilostomídeos, o teste de Friedman $\left(x_{r}^{2}=117\right)$ identificou diferença significativa pelo menos no que se relaciona com uma das técnicas, a nivel de $1 \%$; da comparação múltipla derivaram estas cifras: $\mathrm{Kato}-\mathrm{Katz}=262,5$; McMaster $=228,5$; Stoll-Hausheer $=211(\mathrm{c}=36,6)$.

\section{DISCUSSÃO}

Com base nos objetivos propostos, parece-nos lícito admitir sobre a especificidade:

Para ancilostomideos, favoritismo para a técnica de Kato-Katz, seguida pela de McMaster, revelando-se a de Stoll-Hausheer, surpreendentemente, a menos eficiente;

Para A. lumbricoides, T. trichiurus e \$. mansoni o método de Kato-Katz apresentou também a maior positividade, seguido pelos demais, que no caso particular do $T$. trichiurus mostraram-se equivalentes; no referente a $S$. mansoni e ovos férteis e inférteis de A. lumbricoides, o de McMaster atuou de forma consideravelmente inferior, de modo a não suscitar cogitação quando visado o diagnóstico da esquistossomose mansônica, isso encontrando eventual explicação na alta densidade dos ovos em tela e no fato da técnica ter na flutuação o seu fundamento;

Para $H$. nana, o processo de Kato $-\mathrm{K} t z$ agiu de maneira pouco efetiva, provavelmente pela rapidez com que os ovos pertinentes deixam de ser visualizados.
A análise estatística, à qual submetemos as contagens, permitiu estabelecer as conclusões adiante expostas:

Para ovos de ancilostomideos, os métodos podem ficar escalonados de modo a admitir-se sensibilidades equivalentes entre si, respectivamente para os de Stoll-Hausheer e McMaster e para os de McMaster e Kato-Katz, porém com diferença significativa a favor do de Kato-Katz sobre o de StollHausheer;

Para ovos de T. trichiurus, a técnica de Kato Katz foi a mais sensivel e as demais afiguraram-se inferiores e equivalentes;

Para ovos de $A$. lumbricoides e $S$. mansoni, os processos situaram-se nesta ordem decrescente de sensibilidade: Kato-Katz, Stoll-Hausheer e McMaster;

Para ovos de $H$. nana, as técnicas apresentaram-se semelhantes no que diz respeito à capacidade de detectar maiores números.

Em apreciação global, valorizando, acima de tudo, a padronização em laboratório de análises clínicas, é correto deduzir que o método de Kato-Katz encerra vantagens se consideradas a especificidade e a sensibilidade, ressalvando-se a presença de ovos cuja visualização è extremamente fugaz nas preparações. Vale a pena somar a esses méritos outras virtudes, ligadas à facilidade de manipulação e possibilidade de armazenamento das preparações.

A técnica de McMaster, eletivamente usada em exame de fezes de herbivoros, deixou clara a dificuldade para apreciação microscópica de matéria fecal humana, rica em detritos; ela consubstanciou razoável utilidade à evidenciação de ovos considerados leves, mas portou-se deficientemente quando participavam os pesados.

\section{SUMMARY}

Three hundred stool examinations were performed in order to compare the results of the following techniques: Kato-Katz, Stoll-Hausheer and McMaster.

For helminthic disease the number of positive samples with the Kato-Katz, Stoll-Hausheer and McMaster techniques were as follows: hoowkorm disease $-117(89,7 \%, 76 \%$ and $80.3 \%)$, infection 
Castilho VLP, Guizelini E, Turri ES, Campos R, Amato Neto V, Moreira AAB, Pinto PLS. Exame parasitológico quantitativo das fezes: estudo comparativo entre os métodos de McMaster, Stoll-Hausheer e Kato-Katz. Revista da Sociedade Brasileira de Medicina Tropical 17:209-212, Out-Dez, 1984

due to Trichocephalus trichiurus - $124(91.1 \%$, $70.9 \%$ and $70.9 \%$ ); Ascaris lumbricoides - 59 (94.9\%, 84.7\% and $49.1 \%)$; Schistosoma mansoni$58(96.5 \%, 48.2 \%$ and $0 \%$ ); Hymenolepis nana -15 $(46.6 \%, 73.3 \%$ and $86.6 \%)$.

On statistical analysis the Kato-Katz technique was the most sensitive for faecal egg counts. The Kato-Katz technique is also considered as the most specific and the easiest to perform. The McMaster technique should not be used as a routine procedure, because of its low efficacy in revealing heavy eggs and due to the fact that the samples obtained with this method are difficult to examine.

Key words: Quantitative stool examinations. Comparative trial. Kato-Katz, Stoll-Hausheer and McMaster technique.

\section{AGRADECIMENTO}

Agradecemos à Sra. Sueli da Rocha, Bacharel em Estatistica pela Universidade de São Paulo, pela colaboração prestada à análise estatística dos resultados.

\section{REFERÊNCIAS BIBLIOGRÁFICAS}

1. Amato Neto V, Corrèa LL. Exame parasitológico das fezes. Savier, São Paulo, 1980.

2. Chaia G, Chaia ABQ, McAullife J, Katz N, Gasper D. Coprological diagnosis of shistosomiasis. II -Comparative study of quantitative methods. Revista do Instituto de Medicina Tropical de São Paulo 10: 349-353, 1969.
3. Chaves A, Alcantara OS, Carvalho OS, Santos JS. Estudo comparativo dos métodos coprológicos de Lutz, Kato-Katz e Faust modificado. Revista de Saúde Pública, São Paulo 13: 348-352, 1979.

4. Coura JR, Conceição MJ. Estudo comparativo dos métodos de Lutz, Kato e Simões Barbosa no diagnóstico coprológico da esquistossomose mansoni. Revista da Sociedade Brasileira de Medicina Tropical 8: 153-158, 1974.

5. Gibbons JD. Nonparametric methods for quantitative analyses. Holt, Rinehart and Winston, New York, 1976.

6. Gordon HMcL, Whitlock HV. A new technique for counting nematode eggs in sheep faeces. Journal of the Council for Scientific and Industrial Research 12: 50-53, 1939.

7. Instituto de Pesquisas Johnson e Johnson de Doenças Endêmicas. Metodologia para avaliação terapêutica de drogas antiparasitárias. 1 - Parasitas intestinais. Primeiro Encontro de Pesquisadores em Medicina Tropical, São José dos Campos, São Paulo, 30 de novembro de 1973.

8. Katz N, Chaia G. Coprological diagnosis of schistosomiais. I - Evaluation of quantitative techniques. Revista do Instituto de Medicina Tropical de São Paulo 10: 295-298, 1968

9. Mello DA, Luz PE, Chiarini C. Comparação dos métodos coprológicos quantitativos de Barbosa (1969) e Kato-Katz et al. (1972). Revista Goiana de Medicina 23: 53-59, 1977.

10. Molina JG. A técnica de Kato para o diagnóstico coprológico de infeç̧ões parasitárias. Revista da Sociedade Brasileira de Medicina Tropical 5: 17-20, 1974. 